\title{
MULTI-LABEL CLASSIFICATION FOR DRILL-CORE HYPERSPECTRAL MINERAL MAPPING
}

\author{
I. C. Contreras*, M. Khodadadzadeh, R. Gloaguen \\ Helmholtz-Zentrum Dresden-Rossendorf (HZDR), Helmholtz Institute Freiberg for Resource Technology, \\ Chemnitzer Str. 40, 09599 Freiberg, Germany - (i.contreras, m.khodadadzadeh, r.gloaguen)@ hzdr.de
}

\section{Commission III}

KEY WORDS: Mineral mapping, drill-core hyperspectral data, mineral liberation analysis, classifier chains, random forest, multilabel classification, machine learning.

\begin{abstract}
:
A multi-label classification concept is introduced for the mineral mapping task in drill-core hyperspectral data analysis. As opposed to traditional classification methods, this approach has the advantage of considering the different mineral mixtures present in each pixel. For the multi-label classification, the well-known Classifier Chain method (CC) is implemented using the Random Forest (RF) algorithm as the base classifier. High-resolution mineralogical data obtained from Scanning Electron Microscopy (SEM) instrument equipped with the Mineral Liberation Analysis (MLA) software are used for generating the training data set. The drillcore hyperspectral data used in this paper cover the visible-near infrared (VNIR) and the short-wave infrared (SWIR) range of the electromagnetic spectrum. The quantitative and qualitative analysis of the obtained results shows that the multi-label classification approach provides meaningful and descriptive mineral maps and outperforms the single-label RF classification for the mineral mapping task.
\end{abstract}

\section{INTRODUCTION}

Drill-cores are cylindrical rock samples extracted by drilling from the Earth's subsurface up to hundreds of meters during the exploration campaigns. They represent unaltered samples of rocks and provide valuable information of the subsurface (Gandhi, Sarkar, 2016). Mining companies rely on extracting drill-cores to describe geological systems and target the important ore accumulations in the deposits.

The first task in the drill-core analysis is usually core logging. It consists of describing and recording mineralogical information, such as lithology or alteration patterns, by an expert on-site. By performing core logging on the cores, important regions can be identified which are then sent to laboratories for the implementation of analytical techniques. These laboratory methods include optical microscopy (Krahenbuhl et al., 2015), X-ray diffraction (XRD) (Fox et al., 2016), X-ray Fluorescence (XRF) (Nikonow, Rammlmair, 2017), Scanning Electron Microscopy (SEM) analysis integrated with the Mineral Liberation Analysis (MLA) (Fandrich et al., 2007) or with the QEMSCAN (Gottlieb et al., 2000) software, amongst others.

The mining industry is gradually integrating the use of hyperspectral sensors to complement drill-core analyses. Hyperspectral scanners provide a fast, non-destructive, and non-invasive mean to analyze the cores. Hyperspectral data are recorded in hundreds of spectral bands covering contiguous and narrow wavelengths along the electromagnetic spectrum (Kruse, 1996, van der Meer, 2004). Depending on the sensor, the data can be recorded in the visible-near infrared (VNIR, $350-1000 \mathrm{~nm}$ ), the short-wave infrared (SWIR, $1000-3000 \mathrm{~nm}$ ) or the longwave infrared (LWIR, 8000 - $15000 \mathrm{~nm}$ ). Minerals spectral responses are distinct and mainly related to the fundamental electronic and vibrational process of the molecular bonds. Also,

\footnotetext{
* Corresponding author.
}

the mineral mixtures and different grain sizes have an influence on the spectral signature (Clark, 1999). These distinct spectra helps recognizing a range of minerals.

Classic methods to analyze drill-core hyperspectral data consist of the visual comparison of the general shape and absorption features present in the spectra with a reference spectral library (Huntington et al., 2006, Mauger, Hore, 2009). Another common approach is to use an established chain of techniques available in the well-known software called ENVI (Environment for Visualizing Images. Exelis Visual Information Solutions, Boulder, Colorado). This chain includes the reduction of the dimensionality using a minimum noise fraction transformation, the selection of representative samples or endmembers using the pixel purity index and the n-Dimensional visualizer, a manual or automatic identification of the endmembers using spectral libraries and the spectral analyst, mapping the minerals and/or determining their partial abundances using spectral similarity measure (e.g., spectral angle mapper) or unmixing (e.g., linear unmixing, mixture tune matched filtering) algorithms (Littlefield, Calvin, 2014, Kruse et al., 2012, Calvin, Pace, 2016, Littlefield et al., 2012, Xu et al., 2011, Bedini et al., 2009, Kratt et al., 2010).

Lately, the use and development of machine learning methods for data analysis are growing in different research fields. Machine learning techniques offer automatic approaches to discover patterns and underlying relations within large data sets. More recently, machine learning methods have been also suggested for the analysis of drill-core hyperspectral data to improve the robustness and automation of the analyses (Schneider et al., 2014, Contreras et al., 2019, Tusa et al., 2019, Contreras Acosta et al., 2019b, Wang et al., 2017). However, developing supervised machine learning frameworks for mineral mapping in drill-core hyperspectral data is a challenging task. This is because little prior information can be obtained in a represent- 
ative form to define meaningful classes and select characteristic samples for training. In (Contreras Acosta et al., 2019a), an approach where high-resolution mineralogical data, obtained from polished thin sections of some drill-core samples, are spatially re-sampled and co-registered to the corresponding hyperspectral data was proposed. The co-registered data were then used to generate the reference or ground truth data for training a supervised classification algorithm.

Based on the traditional classification concept each pixel is represented by a single instance (feature vector) and associated with a single label. Thus, the assumption is that each pixel has only one unique semantic meaning/label (Zhang, Zhou, 2014). However, although traditional classification algorithms are highly implemented and performing well, there are many learning tasks where the assumption is not completely suitable, for example in drill-core hyperspectral mineral mapping. This is because of the nature of the drill-core hyperspectral data where the pixels are usually highly mixed and it is difficult to find pure mineral spectra at the current spatial resolution of the data. Thus, pixels in drill-core hyperspectral data are expected to be labeled more meaningfully by giving multiple labels.

The multi-label classification concept was first introduced for text classification (Tsoumakas, Katakis, 2007). In multi-label classification, each pixel is associated with a set of labels $Y \subseteq l$, in contrast to single-label classification where a pixel is characterize by a single label $l$ from a set of disjoint labels $L$ (Tsoumakas, Katakis, 2007, Zhang, Zhou, 2014). In general, the available methods for multi-label classification can be grouped in two main categories: problem transformation methods and algorithm adaptation methods. The first category solves the multi-label problem by transforming it into one or more singlelabel classification problems, whereas the algorithms belonging to the second group focus on extending popular learning techniques to handle multi-label data directly (Tsoumakas, Katakis, 2007, Zhang, Zhou, 2014). The problem transformation methods try to fit data to algorithm, while the algorithm adaptation methods aim at fitting algorithm to data (Zhang, Zhou, 2014).

In this work, we introduce the use of multi-label classification to map minerals in drill-core hyperspectral data. More specifically, for the first time to map minerals, we apply a multilabel classification method, that belongs to the family of problem transformation methods, called classifier chain model (CC) (Read et al., 2009). Broadly, this method links together binary classifiers in a chain where class label predictions become features for the following classifiers (Read et al., 2019). Thus, label correlations are considered. This has more meaning in the case of drill-core hyperspectral data since pixels are highly mixed and certain mineral associations are expected. To be able to implement this method, we use high-resolution mineralogical data (i.e., SEM-MLA) to annotate the hyperspectral pixels in a small area of a drill-core. Pixels in the SEM-MLA are of $3 \mu \mathrm{m}$ size while in the hyperspectral data pixels are of about $1.5 \mathrm{~mm}$. 250000 pixels of the SEM-MLA image comprise 1 pixel at the hyperspectral spatial resolution. Multiple labels are assigned to each pixel in the SEM-MLA region based on the relative abundance of the different minerals. As a base classifier for the CC method, the well-known random forest (RF) algorithm is implemented since it has been shown to provide good results in geological applications and it can handle high-dimensional data with a limited number of training samples (Breiman, 2001).

The rest of the paper is structured as follows: section 2 describes the classifier chain method. Section 3 presents data de- scription and acquisition, experimental results, and discussions. Finally, the conclusions are drawn in Section 4.

\section{FRAMEWORK FOR THE MULTI-LABEL CLASSIFICATION}

In this study, we propose to use the CC method with the RF algorithm as a base classifier to perform the multi-label classification in drill-core hyperspectral data. Once the high-resolution mineralogical data are collected by the SEM-MLA technique, they are linked to the corresponding hyperspectral data (Contreras Acosta et al., 2019a). Then, the labels for each hyperspectral pixel in the SEM-MLA region are chosen based on the frequency of the existing minerals in the SEM-MLA for the area of that pixel. For this, each hyperspectral pixel is labeled with the minerals that represent around $95 \%$ of the content in the SEM-MLA for that pixel.

\subsection{The Classifier Chain (CC) method}

The CC algorithm transforms the multi-label problem into a chain of binary classification problems. Binary classifiers in the chain are built upon the predictions of the preceding ones (Zhang, Zhou, 2014). This is, a chain of classifiers, $C_{1}, \cdots, C_{|L|}$, where each classifier, $C_{j}$, learns and predicts the binary association of label $l_{j} \in L$, given the feature space augmented by all prior binary relevance predictions in the chain $l_{1}, \cdots, l_{j-1}$. The classification begins at $C_{1}$ by determining $\operatorname{Pr}\left(l_{1} \mid \mathbf{x}\right)$ and every subsequent classifier $C_{2} \cdots C_{|L|}$ predicts $\operatorname{Pr}\left(l_{j} \mid \mathbf{x}_{i}, l_{1}, \cdots, l_{j-1}\right)$. Following this method, the label information between classifiers is passed and therefore, labels correlations are taken into account (Read et al., 2009). As it is expected, the order of the chain influences the results. For more details we refer the readers to (Read et al., 2009).

\subsection{Random Forest (RF)}

The RF algorithm is applied as a base classifier in the CC method. In RF, a set of decision tree classifiers are trained and their individual results are combined through a voting process. This type of classifiers is the so-called ensemble learning classifiers (Breiman, 2001). Each tree gives a unit vote to the most frequent class in the input vector $\mathbf{x}$ and the classification label is allocated to the input vector through a majority vote: $\hat{C}_{R F}=$ majority vote $\left\{\hat{C}_{t}(\mathbf{x})\right\}_{1}^{T}$, where $\mathbf{x}$ is the input vector, $\hat{C}_{t}(\mathbf{x})$ is the class prediction of the $t$ th tree, and $T$ shows the total number of trees (Rodriguez-Galiano et al., 2012). Due to the different decision trees, RF is robust and it is expected to perform more accurately than an individual decision tree classifier.

\section{EXPERIMENTAL RESULTS}

In this section the data description (i.e., acquisition and preprocessing), setup employed for the experiments, and results are presented.

\subsection{Data description}

For the acquisition of the drill-core hyperspectral data an unpolished half of a drill-core sample was used (see the RGB image of the Drill-core sample in Fig. 1). The acquisition was performed with a SisuRock drill-core scanner equipped with an 
AisaFenix VNIR-SWIR hyperspectral sensor. This sensor acquires data that encompass the 380-2500 $\mathrm{nm}$ range of the electromagnetic spectrum. The spectral resolution is $3.5 \mathrm{~nm}$ in the VNIR and $12 \mathrm{~nm}$ in the SWIR with a total of 450 bands. The size of the drill-core hyperspectral image is $33 \times 189$ pixels where each pixel covers an area of $1.5 \times 1.5 \mathrm{~mm}^{2}$. In the preprocessing stage of the data, radiometric and geometric corrections were carried out to correct the sensor-shift and the effect of lenses, respectively. For this, the toolbox presented in (Jakob et al., 2017) was used.

For the training set, high-resolution mineralogical data were acquired from a carbon-coated polished thin section of about 30 $\mu m$ thickness from an specific region of the drill-core sample using the SEM instrument with the MLA software. The region where the thin section was taken is represented by the red rectangle in the RGB image of the drill-core shown in Fig. 1 (see Thin section in Fig. 1). In general, the SEM-MLA analysis consists of defining mineral grains using the back-scatter electron signal received off the sample and defining the mineral phases with X-ray measurements on a closely-space grid. Pixels in the resultant SEM-MLA image are of $3 \mu \mathrm{m}$ size (see SEM-MLA image in Fig. 1). 250000 pixels of the SEM-MLA image comprise 1 pixel at the hyperspectral spatial resolution.

\subsection{Experimental setup}

As explained in Section 2, labels for the re-sampled SEM-MLA pixels were chosen based on the frequency of the minerals for that region in the SEM-MLA image. For this, a threshold of around $95 \%$ of the content, in the SEM-MLA, was considered to avoid minerals with very low frequency. 22 minerals are identified by the SEM-MLA analysis (see legend of the SEM$M L A$ image in Fig. 1). However, in the VNIR-SWIR range of the electromagnetic spectrum not all of these minerals have diagnostic absorption features. For example, minerals like Px (piroxene), Qz (quartz), and Py (pyrite) are represented similarly as flat spectra in this range. For this reason, the minerals without representative diagnostic absorption features in the VNIR-SWIR range were grouped as OMs (other minerals).

For a complete qualitative analysis, a typical multi-class RF algorithm was also used to train a model and then, on a later stage, predict a unique label for each pixel in the entire drill-core hyperspectral image. To train the RF classifier, the SEM-MLA image was also considered, however, for each re-sampled pixel a unique label was given (i,e., the most frequent mineral). For all these implementations, 500 decision trees were used and the number of prediction variables was set equal to the number of input bands.

\subsection{Multi-label classification results}

To implement the multi-label classification algorithm, the order of the chain was set based on the frequency of the minerals in the SEM-MLA image. This is because the nature of the SEM-MLA data that gives the quantity of the minerals, therefore, we can conclude that this is the most relevant order. The reference data used to train the CC are shown in Fig. 2. As can be observed, Wmca (white mica), Bt (biotite), Chl (chlorite), Amp (amphiboles), Gp (gypsum), and $O M s$ are the most dominant minerals present in the re-sampled SEM-MLA. Fig. 3 shows the results of the multi-label classification. Wmca and $G p$ are mostly present in the veins and alteration halos, whereas the matrix is mainly composed of $O M s$ with $C h l, B t$,

\begin{tabular}{c||c}
\hline Classes & Accuracies (\%) \\
\hline OMs & 94.64 \\
Wmca & 66.96 \\
Gp & 86.60 \\
Chl & 81.25 \\
Amp & 91.96 \\
Bt & 93.75 \\
\hline \hline
\end{tabular}

Table 1. Classes accuracies for the Classifier Chain RF classification.

\begin{tabular}{c||c}
\hline \hline Classes & Accuracies (\%) \\
\hline Wmca & 14.29 \\
Gp & 57.14 \\
OMs & 97.96 \\
\hline
\end{tabular}

Table 2. Classes accuracies for the traditional RF classification.

and less amount of Wmca and Amp (amphiboles). By comparing these maps with the high-resolution mineralogical image (SEM-MLA image in Fig. 1), a similar mineralogical distribution can be observed. The SEM-MLA image shows that the matrix in the sample is dominantly composed of $P l$ (plagioclase) partly altered to $W m c a, C h l$, and $B t$. The veins are of two types: $Q z$ and $W m c a$ veins, and $G p, W m c a$, and scarce $P y$ veins. Minerals such as $Q z, P l$, and $P y$ are not mapped individually due to the lack of diagnostic absorption features in the VNIRSWIR, but have been included in the OMs class. Moreover, the mineral map obtained from the classic multi-class RF classification (shown in Fig. 4) highlights the advantages and capabilities of the multi-label approach for the mineral mapping task. This is because of the nature of drill-core hyperspectral data which is having mixed pixels, and when considering one single label per pixel the mapping is generalized. Thus, performing a multi-label classification on drill-core hyperspectral data presents an advantage for mapping the different minerals present in the pixels of the sample.

\section{CONCLUSION}

In this paper, we introduce the use of multi-label classification for drill-core hyperspectral mineral mapping. For this, the CC method together with RF is used. This method links together RF binary classifiers in a chain. This means that each class label is predicted by one classifier and the resultant predictions are included in the feature space for the following classifier. For generating the training set, we used SEM-MLA data, which provides high-resolution mineralogical analysis. From the analysis of the results, we have seen that the multi-label classification approach provides more descriptive and meaningful results than a typical RF classification where the mapping is less detailed.

As part of our future work, we will implement other advance multi-label classification algorithms and compare the performance on larger drill-core hyperspectral datasets for the mineral mapping task

\section{ACKNOWLEDGEMENTS}

The authors would like to thank Robert Zimmermann for the acquisition of the hyperspectral data, as well as Laura Tusa for conducting the SEM-MLA analysis. 

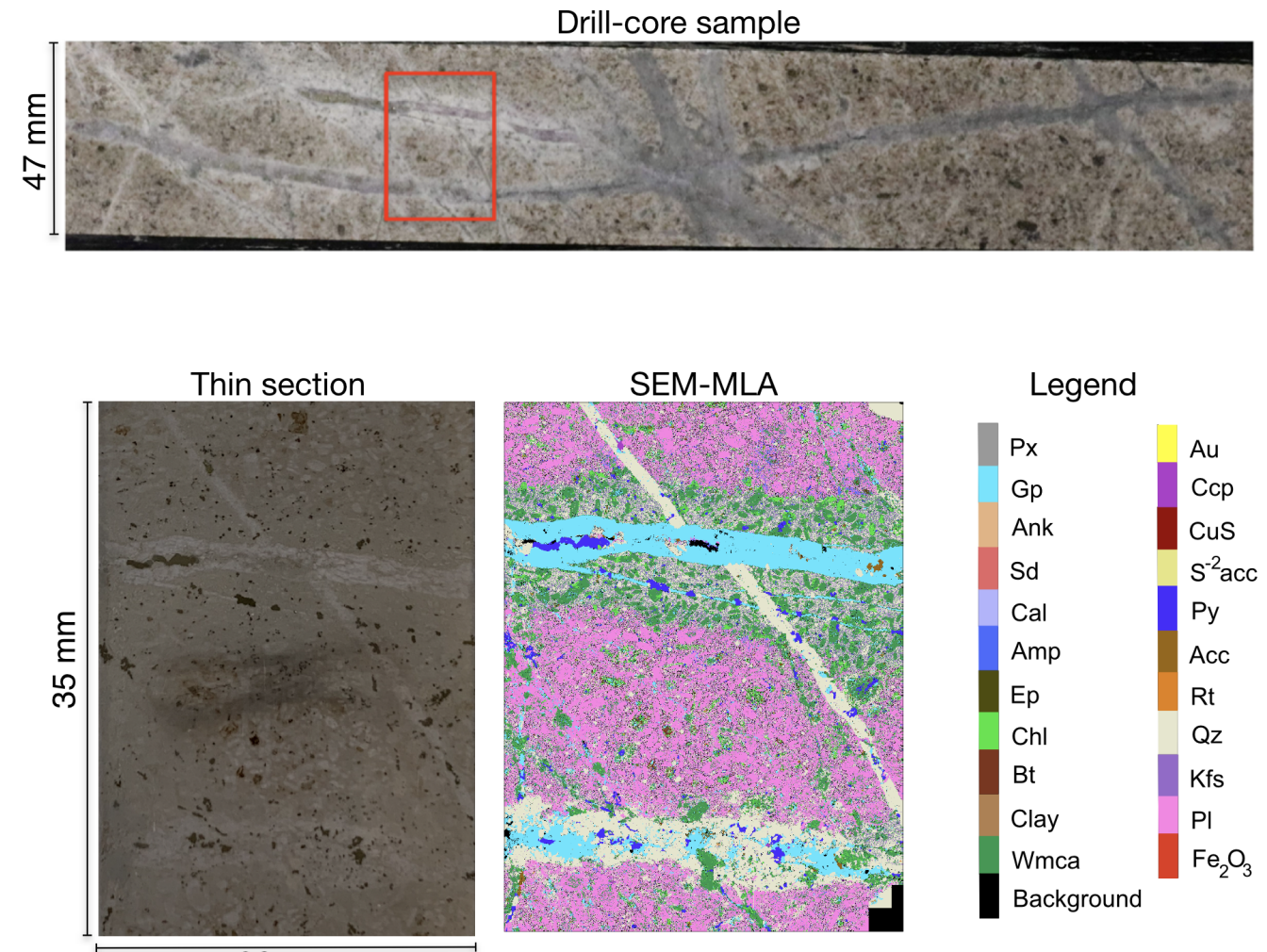

$26 \mathrm{~mm}$

Figure 1. RGB image of the drill-core sample. Thin section of the drill-core acquired from the are shown within the red rectangle in the Drill-core sample, and resultant high-resolution mineralogical image (SEM-MLA image).

Resampled SEM-MLA

Wmca

\begin{tabular}{|c|} 
Amp \\
\hline$\because+2$ \\
$\therefore$ \\
\hline
\end{tabular}

Bt

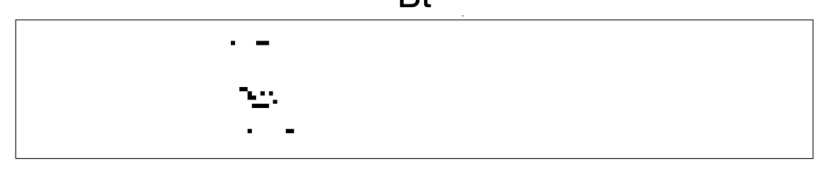

Chl

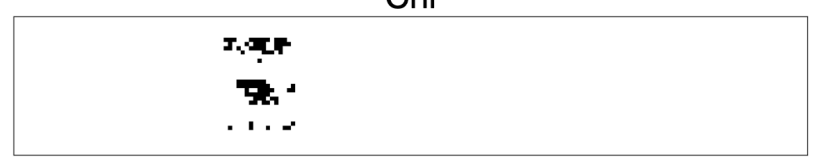

\section{Gp}

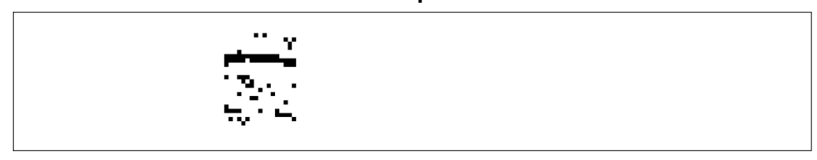

\section{OMs}

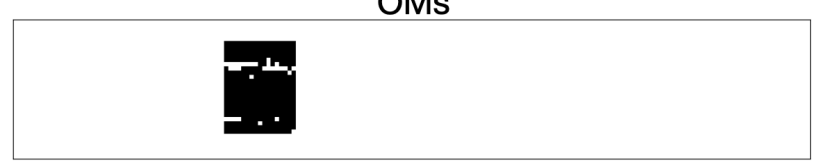

Figure 2. Binary SEM-MLA reference data used to train the Classifier Chain method. 
CC-RF classification

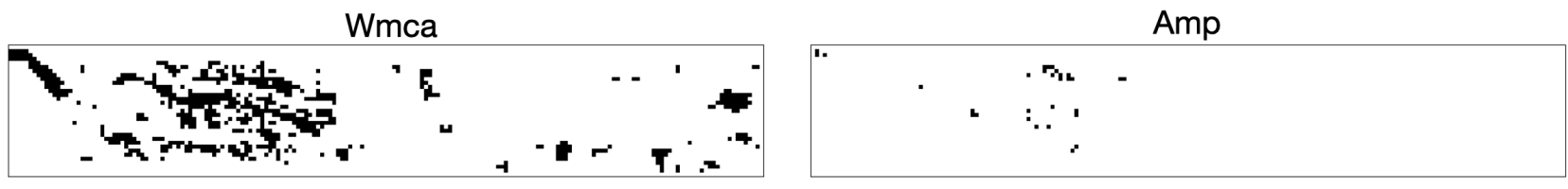

Bt

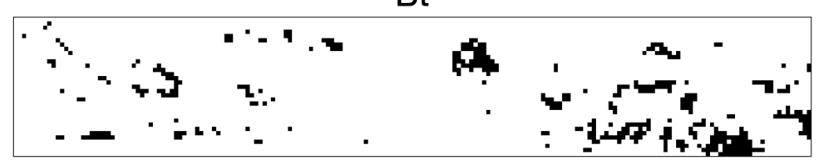

Chl

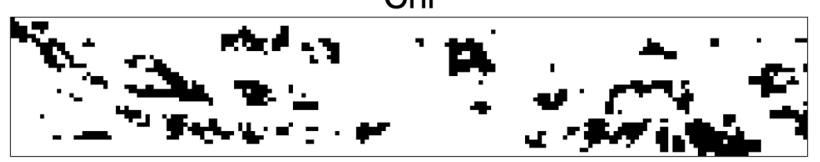

Figure 3. Multi-label classification results of a drill-core hyperspectral image obtained with the Classifier Chain method and a Random Forest algorithm.

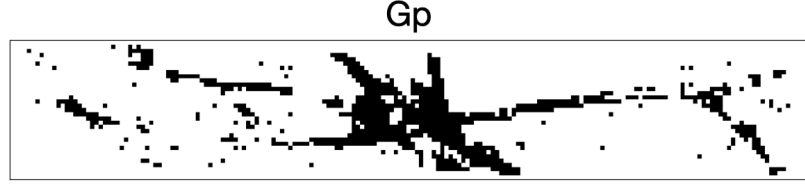

OMs

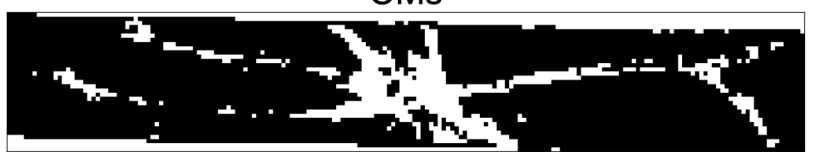

Resampled SEM-MLA

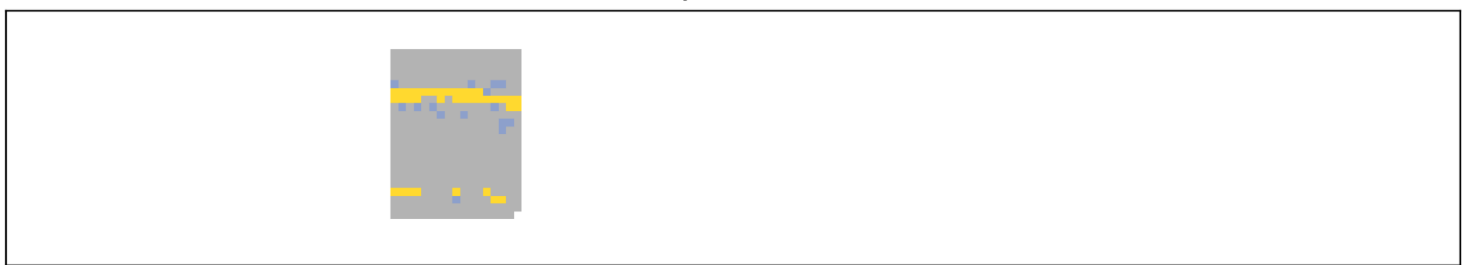

RF classification

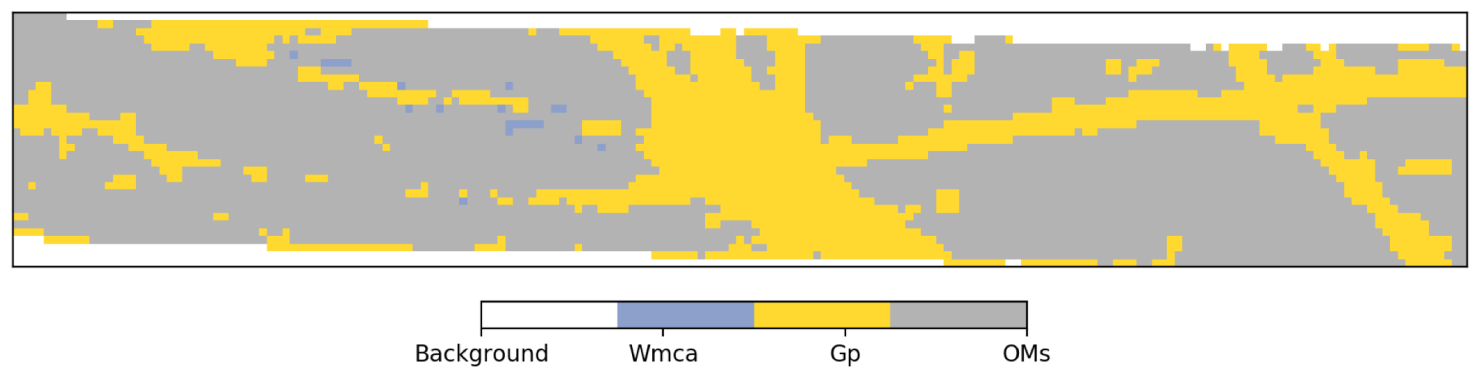

Figure 4. Classification results obtained by Random Forest using as training set the SEM-MLA image considering the most frequent minerals per re-sampled pixel. 


\section{REFERENCES}

Bedini, E., van der Meer, F., van Ruitenbeek, F., 2009. Use of HyMap imaging spectrometer data to map mineralogy in the Rodalquilar caldera, southeast Spain. International Journal of Remote Sensing, 30(2), 327-348.

Breiman, L., 2001. Random Forest. Machine Learning, 45(1), 5-32.

Calvin, W. M., Pace, E. L., 2016. Mapping alteration in geothermal drill core using a field portable spectroradiometer. Geothermics, 61, 12-23.

Clark, R. N., 1999. Spectroscopy of rocks and minerals, and principles of spectroscopy. A. N. Rencz (ed.), Remote Sensing for the Earth Science: Manual of remote sensing, 3, John Wiley \& Sons, Inc, New York, chapter 1, 3-58.

Contreras Acosta, I. C., Khodadadzadeh, M., Tusa, L., Ghamisi, P., Gloaguen, R., 2019a. A Machine Learning Framework for Drill-Core Mineral Mapping Using Hyperspectral and HighResolution Mineralogical Data Fusion. IEEE Journal of Selected Topics in Applied Earth Observations and Remote Sensing, $1-14$.

Contreras Acosta, I., Khodadadzadeh, M., Ghamisi, P., Gloaguen, R., 2019b. Mineral Mapping of Drill Core Hyperspectral Data with Extreme Learning Machines. IEEE Geoscience and Remote Sensing Symposium (IGARSS) 2019, IEEE, 1-4.

Contreras, C., Khodadadzadeh, M., Tusa, L., Loidolt, C., Tolosana-delgado, R., Gloaguen, R., 2019. Geochemical and hyperspectral data fusion for drill-core mineral mapping. 10th Workshop on Hyperspectral Imaging and Signal Processing: Evolution in Remote Sensing (WHISPERS), IEEE, 1-4.

Fandrich, R., Gu, Y., Burrows, D., Moeller, K., 2007. Modern SEM-based mineral liberation analysis. International Journal of Mineral Processing, 84(1-4), 310-320.

Fox, N., Parbhakar-Fox, A., Moltzen, J., Feig, S., Goemann, K., Huntington, J., 2016. Applications of hyperspectral mineralogy for geoenvironmental characterisation. Minerals Engineering, 63-77.

Gandhi, S., Sarkar, B., 2016. Chapter 8 - Drilling. B. S. S.M. Gandhi (ed.), Essentials of Mineral Exploration and Evaluation, Elsevier, 199-234.

Gottlieb, P., Wilkie, G., Sutherland, D., Ho-Tun, E., Suthers, S., Perera, K., Jenkins, B., Spencer, S., Butcher, A., Rayner, J., 2000. Using quantitative electron microscopy for process mineralogy applications. JOM: the journal of the Minerals, Metals and Materials Society, 52(4), 24-25.

Huntington, J. F., Mauger, A. J., Skirrow, R. G., Bastrakov, E. N., Connor, P., Mason, P., Keeling, J. L., Coward, D. a., Berman, M., Phillips, R., Whitbourn, L. B., Heithersay, P. S., 2006. Automated mineralogical core logging at the Emmie Bluff iron oxide- copper- gold Prospect. Mesa Journal, 41, 38-44.

Jakob, S., Zimmermann, R., Gloaguen, R., 2017. The Need for Accurate Geometric and Radiometric Corrections of Drone-Borne Hyperspectral Data for Mineral Exploration: MEPHySTo-A Toolbox for Pre-Processing Drone-Borne Hyperspectral Data. Remote Sensing, 9(1).

Krahenbuhl, G., Hapugoda, P., Warren, K., O’Brien, G., 2015. A new method for obtaining detailed mineral information on individual coal particles at the size that they are used in coke making. Bowen Basin Symposium, 35-40.

Kratt, C., Calvin, W. M., Coolbaugh, M. F., 2010. Mineral mapping in the Pyramid Lake basin: Hydrothermal alteration, chemical precipitates and geothermal energy potential. Remote Sensing of Environment, 114(10), 2297-2304.
Kruse, F. A., 1996. Identification and mapping of minerals in drill core using hyperspectral image analysis of infrared reflectance spectra. International Journal of Remote Sensing, 17(9), 1623-1632.

Kruse, F. A., Bedell, R. L., Taranik, J. V., Peppin, W. A., Weatherbee, O., Calvin, W. M., 2012. Mapping alteration minerals at prospect, outcrop and drill core scales using imaging spectrometry. International Journal of Remote Sensing, 33(6), $1780-1798$

Littlefield, E., Calvin, W., Stelling, P., Kent, T., 2012. Reflectance spectroscopy as a drill core logging technique: an example using core from the Akutan geothermal exploration project. Geotherm. Res. Counc. Trans., 36, 1283-1291.

Littlefield, E. F., Calvin, W. M., 2014. Geothermal exploration using imaging spectrometer data over Fish Lake Valley, Nevada. Remote Sensing of Environment, 140, 509-518.

Mauger, A. J., Hore, S., 2009. Integrating Mineralogical Interpretation of HyLogger Data with HyMap Mineral Mapping,Mount Painter, South Australia. R. K. Jones S. (ed.), Innovations in Remote Sensing and Photogrammetry, SpringerVerlag, Berlin, Heidelberg, chapter Lecture No, 271-280.

Nikonow, W., Rammlmair, D., 2017. Automated mineralogy based on micro-energy-dispersive X-ray fluorescence microscopy ( $\mu$-EDXRF) applied to plutonic rock thin sections in comparison to a mineral liberation analyzer. Geoscientific Instrumentation, Methods and Data Systems, 6(2), 429-437.

Read, J., Pfahringer, B., Holmes, G., Frank, E., 2009. Classifier Chains for Multi-label Classification. Joint European Conference on Machine Learning and Knowledge Discovery in Databases, 1number 1, 254-269.

Read, J., Pfahringer, B., Holmes, G., Frank, E., 2019. Classifier Chains: A Review and Perspectives. 1-19.

Rodriguez-Galiano, V. F., Ghimire, B., Rogan, J., Chica-Olmo, M., Rigol-Sanchez, J. P., 2012. An assessment of the effectiveness of a random forest classifier for land-cover classification. ISPRS Journal of Photogrammetry and Remote Sensing, 67(1) 93-104.

Schneider, S., Murphy, R. J., Melkumyan, A., 2014. Evaluating the performance of a new classifier - the GP-OAD: A comparison with existing methods for classifying rock type and mineralogy from hyperspectral imagery. ISPRS Journal of Photogrammetry and Remote Sensing, 98, 145-156.

Tsoumakas, G., Katakis, I., 2007. Multi-label classification: An overview. International Journal of Data Warehousing and Mining, 3(3), 1-13.

Tusa, L., Andreani, L., Khodadadzadeh, M., Contreras, C., Ivascanu, P., Gloaguen, R., Gutzmer, J., 2019. Mineral mapping and vein detection in hyperspectral drill-core scans: Application to porphyry-type mineralization. Minerals, 9(2), 1-23.

van der Meer, F., 2004. Analysis of spectral absorption features in hyperspectral imagery. International Journal of Applied Earth Observation and Geoinformation, 5(1), 55-68.

Wang, D., Lagerstrom, R., Sun, C., Laukamp, C., Quigley, M., Whitbourn, L., Mason, P., Connor, P., Fisher, L., 2017. Automated vein detection for drill core analysis by fusion of hyperspectral and visible image data. M2VIP 2016 - Proceedings of 23rd International Conference on Mechatronics and Machine Vision in Practice.

Xu, N., Hu, Y.-X., Lei, B., Hong, Y.-T., Dang, F.-X., 2011. Mineral information extraction for hyperspectral image based on modified spectral feature fitting algorithm. Spectroscopy and Spectral Analysis, 31(6), 1639-1643.

Zhang, M. L., Zhou, Z. H., 2014. A review on multi-label learning algorithms. IEEE Transactions on Knowledge and Data Engineering, 26(8), 1819-1837. 